membership, hours spent at the club, number of sports activities at the club, types of activities practiced at the club.

Main Outcome Measurements The main measurement is the self-medication.

Results Almost half of our 338 amateurs (49.7\%) visiting fitness centers admitted to self-medicate in the context of sport practice. Among them $36.4 \%$ take dietary complements and $22.5 \%$ used drugs. The probability of self-medication increases significantly with the number of hours of sports practice, with the participation to body-building group lessons and with the consumption of sports drinks. Furthermore, 20\% of the substances consumed, whether self-medicated or not, contained potentially doping substances.

Conclusions We could highlight a significant prevalence of self-medication among amateur athletes visiting fitness centers. The intensity and frequency of sports practice seems to play a key-role regarding the decision to self-medication. Given the health risks entailed by these practices it seems crucial to implement information and prevention measures regarding self-medication. Moreover, it would be interesting to lead further research assessing the adverse consequences of self-medication among this specific population.

\section{SERUM VITAMIN D LEVELS AND RISK OF MUSCULOSKELETAL INJURY IN UNIVERSITY TRACK AND FIELD ATHLETES}

Timothy Miller, Sarah Harangody, Scott Kuzma, Robert Magnussen. The Ohio State University Wexner Medical Center, Columbus, Ohio, USA

\subsection{6/bjsports-2021-IOC.326}

Background While vitamin D has been shown to be an important factor in muscle, tendon, and bone health, there is limited data on the relationship of serum vitamin D levels and injury risk in otherwise healthy collegiate athletes.

Objective To determine the prevalence of inadequate serum vitamin D levels in Division I collegiate athletes and risk of musculoskeletal injury in relation to vitamin D levels.

Design Retrospective review of injury tracking database records of track and field athletes at our institution was performed to collect age, race, serum vitamin D level, and injury history.

Setting Division I collegiate athletics.

Patients (or Participants) Track and field athletes at our university who underwent serum vitamin D testing between October 2018 - February 2019.

Interventions (or Assessment of Risk Factors) Vitamin D level was measured using serum total 25-hydroxy vitamin D (25$\mathrm{OH}$ vitamin D). Insufficient and deficient values were combined and labeled as 'inadequate' if they were measured to be less than $32 \mathrm{ng} / \mathrm{mL}$. Information was collected for 34 athletes (13 males, 21 females).

Main Outcome Measurements Serum 25-OH vitamin D level and musculoskeletal injury history

Results Of the 34 athletes tested 14 were measured in the inadequate range $(16.4 \mathrm{ng} / \mathrm{mL}$ to $29.4 \mathrm{ng} / \mathrm{mL})$. 6 of 7 female sprinters and all male sprinters $(n=2)$ fell in the inadequate range. Statistically significant correlation was demonstrated between race and vitamin $\mathrm{D}$ level with more black athletes demonstrating inadequate vitamin D levels $(p=.035)$. There was no statistical correlation between athletes sustaining injuries and having inadequate serum vitamin D levels.
Conclusions Among Division I collegiate track and field athletes there was no statistical correlation between inadequate serum vitamin D levels and musculoskeletal injury risk. There was a higher rate of inadequate serum vitamin D levels among black athletes. Future studies with larger numbers of athletes may demonstrate a correlation between low serum vitamin D levels and musculoskeletal injury rate.

\section{KNOWLEDGE, ATTITUDE AND USAGE OF DOPING DRUGS AMONG NATIONAL LEVEL ATHLETES IN SRI LANKA}

Akhila Nilaweera, Upuli Nadishani, Geeth Nipunya, Naveen Wijekoon. Faculty of Medicine, University of Colombo, Colombo, Sri Lanka

\subsection{6/bjsports-2021-IOC.327}

Background Doping incidence has been increased in sports at all levels. Therefore a need for alternative approaches to address the doping concerns has arisen because doping not only threatens the health of athlete but also the true spirit of sport.

Objective To assess knowledge, attitudes and usage regarding doping among national level Sri Lankan athletes and to identify reasons for doping and to plan better doping prevention strategies were the objectives.

Design Cross sectional descriptive study in which self-administered questionnaire was used to assess knowledge and usage of doping drugs. A modified version of Performance Enhance Attitude Scale was used to assess attitudes.

Setting 20 national teams/pools of Sri Lanka for the years 2018/2019

Patients (or Participants) 347 national athletes recruited through stratified and random sampling

Results The majority of athletes $(66.6 \%)$ could not define the term WADA. Not more than $38 \%$ of athletes were aware about any given drug. Though attitudes regarding doping were positive in general, $8.3 \%$ of athletes admitted they would dope if they got a chance while athletes of individual sports had negative attitudes. According to Randomized Response Technique used, $8.4 \%$ of athletes had admitted usage of doping drugs. Doping was highest among athletes in individual sports (18.2\%). Lack of knowledge, lack of self confidence and pressure to win were the main reasons for doping. Younger age, less experience, professionally being only an athlete, negative attitudes and knowing fellow athletes who used doping drugs were significantly associated $(\mathrm{p}<0.05)$ with doping.

Conclusions Younger inexperienced athletes and athletes of individual sports were more prone to doping and authorities should take special care of them. Actions should be taken to fill the knowledge gaps regarding doping. Special attention should be paid to improve positive attitudes regarding doping because negative attitudes were associated with doping than poor knowledge.

\section{THE IMPACT OF SPORT SPECIALIZATION ON INJURY, SUCCESS, AND PERCEPTIONS OF HEALTH}

Julie Agel, Todd Rockwood, Anne Eaton. University of Minnesota, Minneapolis, USA

10.1136/bjsports-2021-IOC.328 